\title{
Reprint
}

\section{A DVB-S2 Signal Generator with Forward Satellite Channel Impairments}

\author{
P. Savvopoulos and T. Antonakopoulos
}

\section{Instrumentation and Measurement Technology Conference IMTC 2006}

SORRENTO, ITALY, APRIL 2006

\begin{abstract}
Copyright Notice: This material is presented to ensure timely dissemination of scholarly and technical work. Copyright and all rights therein are retained by authors or by other copyright holders. All persons copying this information are expected to adhere to the terms and constraints invoked by each author's copyright. In most cases, these works may not be reposted or mass reproduced without the explicit permission of the copyright holder.
\end{abstract}




\title{
A DVB-S2 Signal Generator with Forward Satellite Channel Impairments
}

\author{
Panayiotis Savvopoulos and Theodore Antonakopoulos \\ Department of Electrical Engineering and Computers Technology, \\ University of Patras, 26500 Rio, Patras, Greece, \\ Phone: +30-2610-996-487, Fax: +30-2610-996-834, \\ E-mail: \{psavvop, antonako\} @upatras.gr.
}

\begin{abstract}
This paper presents a flexible and adaptable instrument for generating DVB-S2 signals for multi-level testing and performance analysis of satellite receivers. The instrument is based on a high level system model that describes and implements the processing chain of the DVB-S2 transmission system and GEO satellite channel impairments insertion mechanisms, while it utilizes high-speed and high resolution DACs for output signals generation. With the contribution of such a setup, numerous validation and verification tests, under real conditions, can be performed on prototype implementations and realization development stages of emerging DVB-S2 satellite receivers.
\end{abstract}

Keywords - Programmable Instrument, Signal Generator, DVB-S2 Standard, Forward GEO Satellite Channel Impairments.

\section{INTRODUCTION}

During the last decade several achievements have taken place in the field of digital communications and more specifically on error correcting codes and modulation techniques. The capabilities, derived from the use of new error correcting schemes, have opened new paths for more effective and more reliable communications, even in 'hostile' channels. Meanwhile, the growing demand of satellite network operators and consumers for new applications and services, over wide bandwidth satellite transponders, led to the definition of the second generation standard for broadband satellite communications, DVB-S2 [1]. The aim of the new standard was not to substitute the previous and globally deployed DVB-S, but to enhance its capabilities and potential during a transitional period of coexistence [2], [3].

The specification of DVB-S2 was made for high performance broadcasting, which is related to power and spectral efficiency, adaptivity/flexibility at reasonable receiver complexity. Based on these features, DVB-S2 is capable of meeting today's satellite broadcasting needs and demands for effective exploitation of available resources on space segments. All of these characteristics are based on the versatility provided by the DVB-S2 physical layer, due to a variety of modulation and coding schemes that are able to change on a frame-by-frame basis, according to channel conditions [1], [4].
Due to the characteristics and peculiarities encountered in the forward GEO satellite channel, such as low SNR operation, non-linearities, weather/atmosphere impairments e.t.c., the design of efficient DVB-S2 receivers turns to be a great challenge. This becomes worse by the decision of the DVB development group to retain the low cost outdoor units of the employed DVB-S terminals, during the transition from the older standard to the new one. Gradually, considerable activity is observed in the field of synchronization mechanisms performed in such digital receivers. This effort seeks for a generic and effective solution suitable for all different DVB-S2 transmission modes, given that existing conventional techniques currently applied, seem to be incapable of coping with the arised conditions [5]. As a consequence, there is an increasing need for a versatile and flexible instrument that is able to provide the necessary signal (continuous or discrete) for multi-level verification, validation and performance evaluation of different DVBS2 compliant receiver implementations and signal processing techniques.

This work aims to fulfil the need of such an intelligent signal generator, in an effective test-bed, for new emerging receiver designs and realizations. The proposed instrument is based on a computing device that hosts a high level system model along with a powerful platform including high-speed and accuracy DACs. The model, which was developed in Matlab/Simulink, consists of two basic components, the DVB-S2 transmission and the forward GEO satellite channel impairments submodules. The instrument is capable of generating either the digital signal samples derived at the output of the ADCs of a digital receiver or the respective complex envelop signal waveforms at an IF frequency that reaches the digital receiver's ADC.

Section II gives an overview of the DVB-S2 Signal Generator architecture and describes its different submodules. In Section III the description of the DVB-S2 transmission system model is presented, while Section IV highlights the forward satellite channel impairments model applied to the generated DVB-S2 signal, including transmitter and receiver ODUs impact. Finally Section V presents the instrument's operational modes along with performance measurements. 


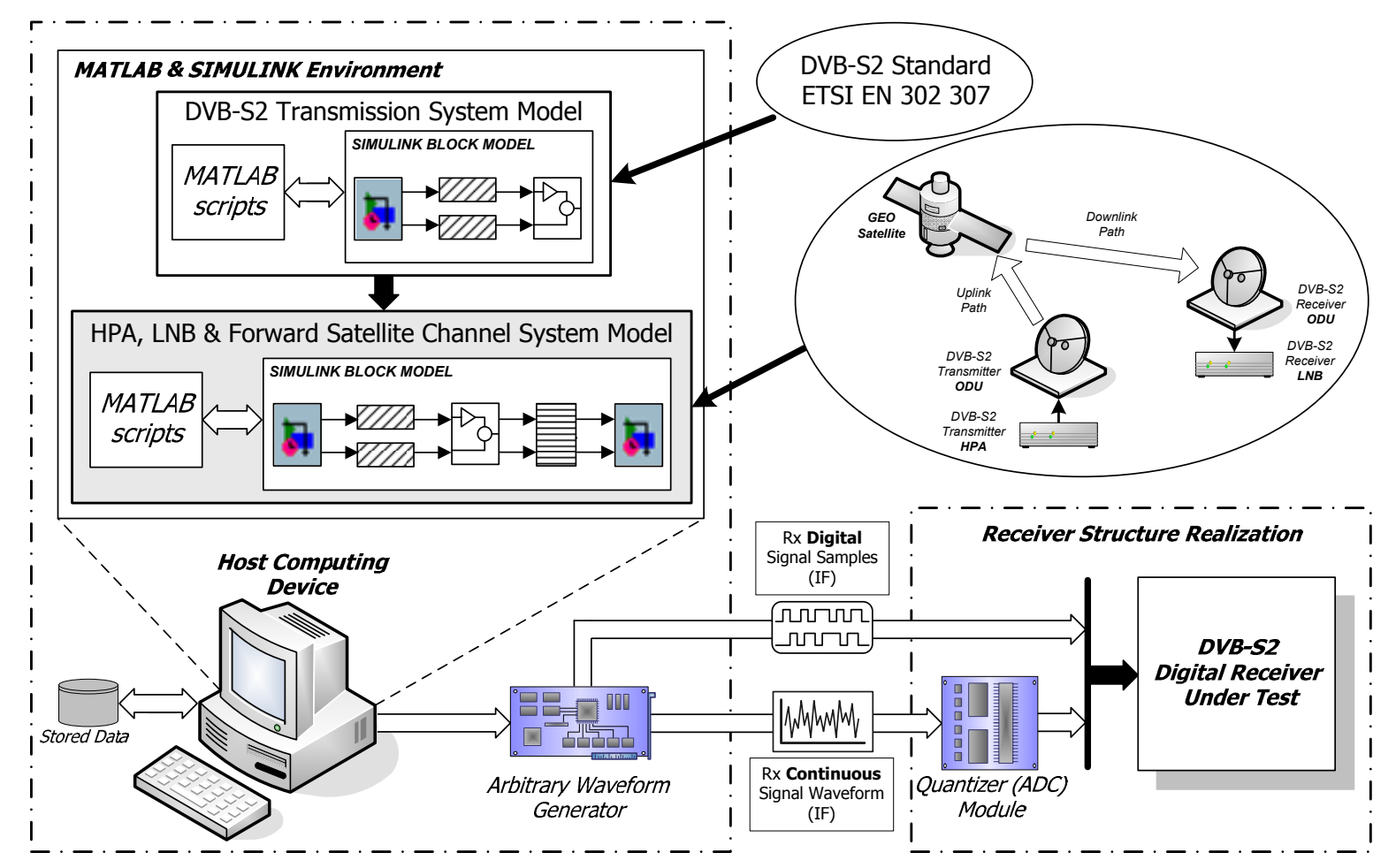

Fig. 1. The DVB-S2 Signal Generator.

\section{THE SIGNAL GENERATOR'S STRUCTURE}

The signal generator consists of a typical computing device along with a high-speed and high-accuracy arbitrary waveform generator platform. The computer hosts the analytical highlevel system model of the DVB-S2 transmission system along with the impairments model, encountered up to the input of a digital receiver. The DVB-S2 transmission system model is responsible for generating a DVB-S2 frame sequence of variable length, modulation and coding modes. Such a sequence corresponds to a signal that is transmitted over the channel, based on a GEO satellite transponder. The term 'channel' incorporates all noise sources that interfere between the transmitter and receiver ends. Consequently, the channel impairments model includes antennas, ODUs (Tx HPA- Rx LNB) of both communicating devices, a complete model of a GEO bend-pipe satellite transponder along with the corresponding descriptions of the up and downlink paths of the forward satellite channel.

The model generates digital samples that can be properly used for generating analog orthogonal waveforms or the respective digital samples, both representing the complex envelop signal at an IF frequency reaching a digital receiver. In the first case the generated signal corresponds to analog waveforms that feed the ADCs of a digital receiver. The second case represents the digital samples, derived from the ADC submodule, that feed the following processing stages of a corresponding digital receiver.

The two analog outputs of the instrument are generated using high-speed DACs with up to $160 \mathrm{MSamples} / \mathrm{sec}$ input data rate, which under interpolation, can reach 400 MSamples/sec. The option of selecting either analog or digital output signals declares the flexibility of the proposed instrument to provide the suitable DVB-S2 signal for various receiver implementations and realizations. Figure 1 gives an overview of the presented instrument.

Due to many parameters and configurations of the instrument submodules, a user friendly interface was developed in Matlab/Simulink environment for their setting-up. Using this interface we are able to define not only model parameters such as transmission parameter scenario (frame length, payload modulation and coding), physical dimensions and noise level fluctuation scenario (antenna diameters, RF frequencies for up and down links, distances, rain rates, phase noise e.t.c) but to determine the DACs sampling rate and interpolation mode. The available modes of operation are analyzed thoroughly in Section V.

\section{DVB-S2 TRANSMISSION SYSTEM MODEL}

The DVB-S2 transmission system model is shown in Figure 2 as a sequence of functional blocks. The signal generation is based on the concept of three framing types:

- BBFRAME derives from the 'Mode \& Stream Adaptation' blocks of the DVB-S2 transmission system that are extensively described in [1], [2], [4]. The model blocks mainly provide input stream interfacing and the required signalling and tools for receiver configuration. Additionally, they packetize the input data streams into requisite 


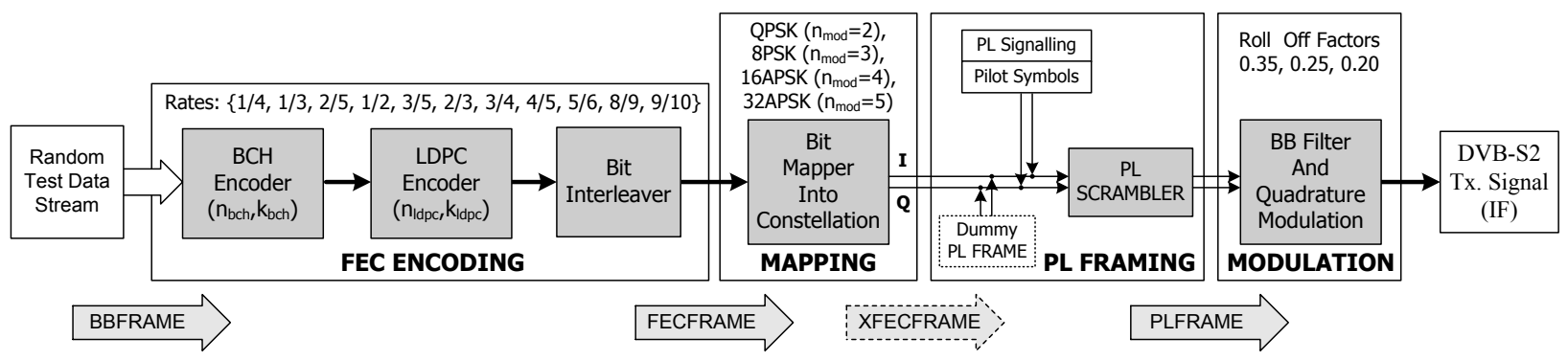

Fig. 2. Block diagram of the DVB-S2 Transmission System Model.

data blocks for proper processing by following FEC submodules.

- FECFRAME includes the concatenation of $\mathrm{BCH}$ and LDPC codes. At its output a bit interleaver is applied when constellation of 8PSK, 16APSK or 32APSK is used.

- PLFRAME carries few highly protected signalling bits over the physical layer and adds specific fields in the transmitted signal to ensure robust synchronization and signalling. It consists of data mapping into constellation procedures, PL header generation, optional Pilot Symbol and Dummy PL frame insertion.

After PL framing, the pulse shaping and quadrature modulation takes place. In this work we focused on the procedures performed from the level of FEC encoding to baseband filtering and quadrature modulation, which remain independent of the supported application and strongly affect the physical layer performance.

\section{A. FEC Encoding}

The DVB-S2 forward error correction (FEC) encoding encompasses three components: an outer systematic $\mathrm{BCH}$ code, an inner LDPC block code and a block bit interleaver. The FEC encoding block receives BBFRAMEs and outputs FECFRAMEs.

Initially the input BBFRAME is encoded by a $\mathrm{BCH}$ code. The introduced outer $\mathrm{BCH}$ codes are specified by the uncoded input block length $k_{b c h}$, which is related to the rate of the used LDPC code [1]. At the BCH output, the $n_{b c h}$ bits are further LDPC encoded. Linear LDPC block codes encode a block of $k_{l d p c}$ information bits to a codeword of size $n_{l d p c}$ bits. It is worth to mention that DVB-S2 systems define and use LDPC codes that are irregular and enable fast systematic encoding.

The total block length of the concatenation of $\mathrm{BCH}$ and LDPC codes is 64800 bits for normal and 16200 for short frame length. The rates of $1 / 4,1 / 3,2 / 5,1 / 2,3 / 5,2 / 3,3 / 4$, $4 / 5,5 / 6,8 / 9,9 / 10$ are supported, with some exceptions according to the used constellation. FEC mode is always constant inside a frame but can change in a frame-by-frame basis. The output codewords are then bit-by-bit interleaved with a block interleaver, where the input data are written into the interleaver column-wise and read out row-wise.

\section{B. Bit Mapping - Constellation}

The mapping submodule utilizes four different constellation diagrams for the mapping of the input bits of a FECFRAME into respective symbols. The utilized diagrams are QPSK, 8PSK, 16APSK and 32APSK with bit efficiencies $n_{\text {mod }}$ ranging from 2 to 5 bits per symbol. A complex XFECFRAME consists of $64000 / n_{m o d}$ and $16200 / n_{\text {mod }}$ symbols for normal and short frame respectively.

\section{PL Framing}

The symbols of the received XFECFRAME are placed in a PL frame that includes slots of 90 symbols each. The XFECFRAME contains a variable number of slots that is related to the modulation format and frame length used for the payload transmission. The PLFRAME derives from the addition of the PL header that represents a slot containing synchronization and PL signalling information from which the receiver is informed about the coding and modulation parameters of the following payload after its successful decoding.

The first 26 binary symbols of the header correspond to a fixed bit sequence that is used by the receivers for start of frame (SOF) detection. The remaining 64 symbols contain information about the system configuration. Due to the critical information contained in the above symbols, the respective bits are highly protected using a pair of interleaved first-order ReedMuller block codes. Finally, the PLS codeword is scrambled by a 64-bits $\mathrm{m}$-sequence. The conversion of the $90 \mathrm{PL}$ header bits into transmission symbols is performed using $\pi / 2$-BPSK modulation.

Concluding, the PL framing provides the insertion of dummy PL frames, when no useful data are ready for transmission and the insertion of optional pilot symbols among payload slots. The pilot symbols comprise an unmodulated carrier as they are characterized by the equality between their in-phase and quadrature components and they are grouped in fields of 36 symbols. The frequency of appearance is every 16 payload slots. The derived sequence of symbols is afterwards scrambled for energy dispersal with a Gold sequence that can vary between different services in the same transponder, or different transponders, for rapid identification of independent data streams. 


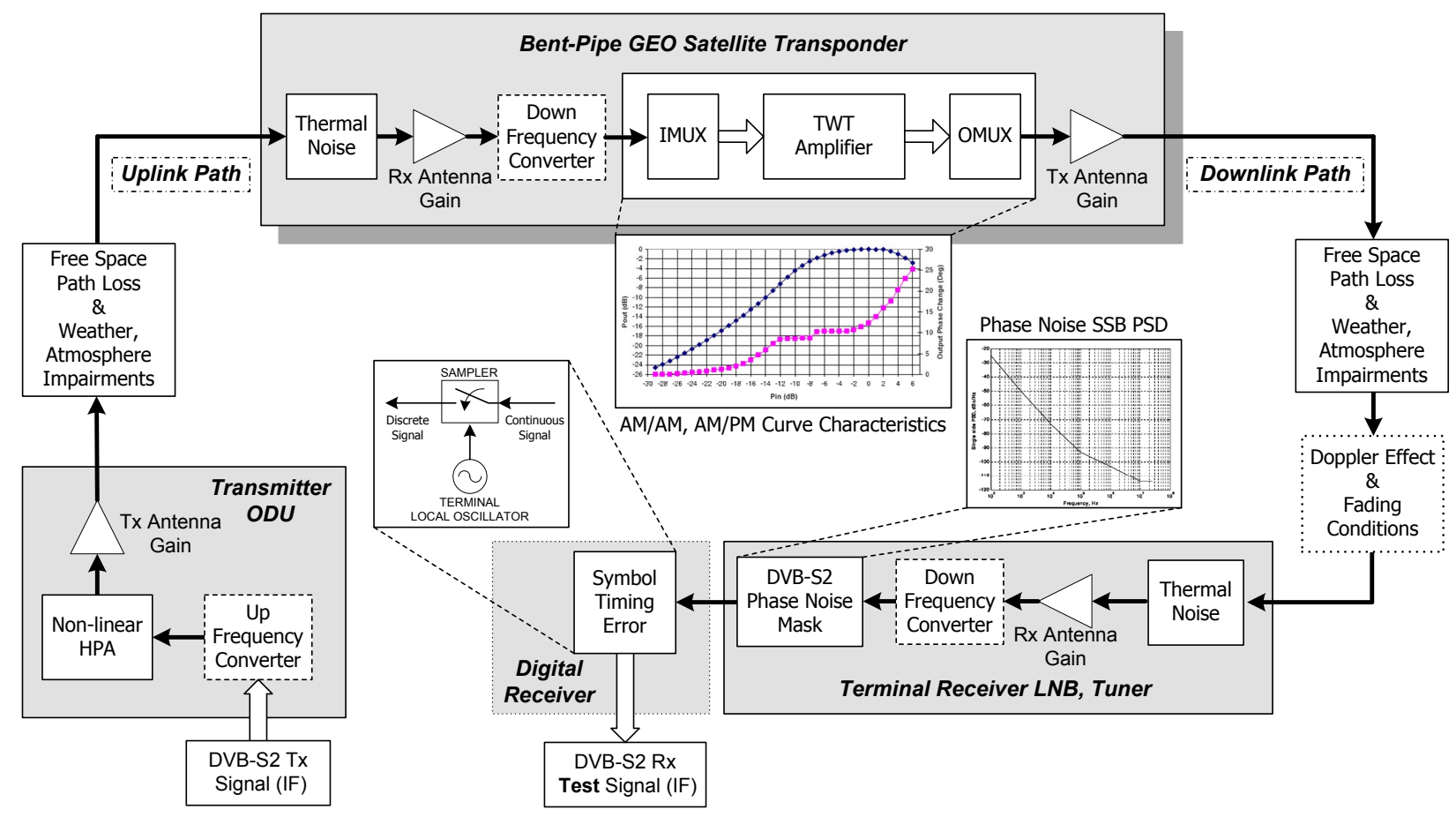

Fig. 3. Block diagram of the Impairments and Forward Satellite Channel Model.

\section{Baseband Shaping and Quadrature Modulation}

The final processing of the DVB-S2 physical layer includes a square root raised cosine filtering for shaping the signal baseband spectrum. The supported filter roll-off factors are 0.35 , 0.25 and 0.20 . At filter output a quadrature modulation generates the upconverted signal by transferring the signal spectrum to the required IF center frequency.

\section{FORWARD SATELLITE CHANNEL AND ODUS IMPAIRMENTS MODEL}

In this section we deal with the details of the high level model that describes the impairments affecting the physical layer performance, which is defined from the IF modulator output of the transmitter until the ODU and the tuner stages at the remote receiver. Between these ends, except from the typical forward satellite channel that consists of the uplink path, space segment and downlink path, the outdoor units (ODUs) of the transmitter and receiver ends are also included. In the following subsections the various noise sources, related to the various components and locations of the model, are described. A representation of the above model is given in Figure 3.

\section{A. DVB-S2 Transmitter $O D U$}

The DVB-S2 IF signal at the transmitter output feeds the Tx ODU. Initially, the signal is converted to the proper RF frequency by an upconversion stage. Then a non-linear High Power Amplifier (HPA) amplifies the signal and feeds a parabolic dish antenna for transmission over the uplink path. Due to the lack of limits in terms of power consumption, physical size and thus peak power of the earth station amplifier, along with the possibility of relatively large dish diameter, the HPA operational point usually lies in the linear region with the minimal AM/AM and AM/PM conversions. Accordingly, the transmitter is able to provide the necessary radiated power (EIRP) without the need for its HPA to operate near the saturation point (input-back-off $\mathrm{IBO}, 0 \mathrm{~dB}$, maximum power). As a consequence, the non-linear impairments introduced by the Tx ODU, are considered negligible. The upconversion contributes to signal degradation in terms of tuner oscillator phase noise, but its effect is taken into consideration at the receiver's respective modules.

\section{B. Up and Downlink Paths}

The signal generated at the output of the Tx ODU, during its propagation through the uplink path, is attenuated and distorted. The main factors that contribute to the signal degradation are the space path loss and weather/atmosphere induced impairments. The first is related with the distance 'transmittersatellite transponder' and the RF uplink frequency. The second includes the dominant weather and atmosphere phenomena encountered in $\mathrm{Ku}$-band satellite communications systems, rain and scintillation respectively. Rain attenuation fluctuates dynamically and depends on rain rate at the location, RF frequency and elevation angle of the earth station antenna. Regarding scintillation effect, it consists of rapid fluctuations of the amplitude and phase of the signal, being propagated, resulting from refractive index irregularities in the transmission path with time. Both signal degradation causes are modeled as random processes, whose amplitudes are described by properly defined lognormally distributed PDFs. 
The downlink path is characterized by the same peculiarities as the previously mentioned uplink path. According to [6], the Doppler effects, due to the GEO satellite movements and the assumption of motionless remote receiver in the developed model, are negligible. For the above reasons Doppler frequency shifts and fading conditions were not included in the impairments model, which can be later added to support also a mobile receiver. These impairments are depicted in Figure 3 as potentially added submodules.

\section{Bent-Pipe GEO Satellite Transponder}

Transponders comprise major components of GEO bentpipe satellite systems and a representative type of impairment encountered in satellite communications channels. For the needs of this work, a model of a transponder was developed. When the propagated signal reaches the Rx satellite antenna, it is initially downconverted to the proper downlink RF frequency and then feeds the satellite transponder submodules. Transponders are characterized by their bandwidth and the amplification stage based on a Travelling Wave Tube amplifier (TWTA). Bandwidth is defined by IMUX and OMUX filters at the input and output of the amplifier. TWTA is described by non-linear AM/AM and AM/PM curve characteristics, that define the alterations occurred on the amplitude and phase of the amplified signal. In our case, the model of a Hellas-SAT 2, $36 \mathrm{MHz}$ transponder was developed from the respective AM/AM,AM/PM measurement graphs [7], but any other satellite transponder can be supported. Due to its near saturation operation, ensuring large coverage and conversion efficiency, increased adjacent channel interference and warping/clustering of the signal constellation, due to intermodulation products, are observed. Apart from the above impairments, a thermal noise block is also added in front of the transponder Rx antenna in order to simulate the noise induced by the transponder electronic circuits and uplink path transmission. The effect of downconversion operation is also considered at the receiver end side.

\section{DVB-S2 Receiver $L N B(O D U)$ and Tuner Stage}

The rest of the model impairments, are related to the receiver ODU and Rx tuner circuits. Thermal noise is induced by receiver circuits and downlink path transmission and is the first block of the Rx ODU/LNB model. In general, synchronization impairments such as carrier and terminal clock frequency errors acquire relatively slowly varying values in comparison to the information rate. LNB and tuning stages of the receiver ODU play a significant role in the degradation of the predescribed characteristics, since they have poor quality. Carrier frequency errors arise mainly from $L N B$ and Tuner Local Oscillators ( $L N B$-Tuner $L O s$ ) noise in terms of frequency drifts and declinations that militate against the proper downconversion functions (e.g. RF to L band, L to IF band). Such operations are depicted in the frequency downconversion block. Apart from the latter cause, additional frequency conversions that are performed on the satellite transponder and even at the transmitter ODU have their impact on the carrier frequency quality. Given the complicated realization of the oscillators noise, DVB-S2 group defines a phase noise mask that concentrates the dominant contribution of LNB and tuner stages noise of consumer reception systems with other conversions occurring in the entire processing chain [1]. The phase noise is based on the single side band (SSB) PSD description of the carrier with noise. The respective model passes white Gaussian noise through a structure of IIR filters that later multiplies the input signal. Likewise, clock frequency variations, derived mainly from Terminal Local Oscillator (Terminal-LO) instabilities and long term drifting, affect the sampling clock of the ADC module at the receiver demodulator input (Symbol Timing Error block). Doppler effect is considered minimal for both synchronization impairments due to the use of GEO satellite communications systems with motionless remote receivers.

\section{IMPLEMENTATION ISSUES}

The presented instrument incorporates two different functional modes of operation resulting to finite or continuous DVB-S2 signal generation. Signal generation starts with the processing of a number of DVB-S2 frames, by the transmission and impairments insertion submodules of the high-level model. All parameters, regarding both submodules of the model, are previously defined in the form of initialization vectors. These vectors include significant parameters such as symbol rate, number, length and MODCOD modes of sequential frames, noise sources levels, DAC accuracy e.t.c. Afterwards, the model is initiated and generates the respective digital samples based on the characteristics of the waveform generator hardware platform. The model generated samples are stored in a file. Before the initiation of the D/A conversion, the hosting device restores the saved samples and fills the available platform memory with a number of output values. When the D/A conversion (signal generation) is in progress, the host processor monitors the output memory on the platform and provides new samples to the memory.

Due to a variation between the maximum supported data rates at the "host-platform" (PCI bus I/F - 100MB/s) and "platform memory-DACs" (2 Channels of 16bits - up to 160MSps $=640 \mathrm{MB} / \mathrm{s}$ ) interfaces, continuous signal generation is feasible only when the above rates are equal. On the contrary, when the "platform memory-DACs" data rate exceeds the rate at the "host-platform" interface, the above operation has an upper limit. In order to harmonize DACs rate with the rate achieved at the "host-platform" interface, the symbol rate (Baud) of the generated signal waveforms and thus the communication signal spectrum must be reduced. Based on this approach, the evaluated maximum symbol rate that makes the instrument capable of generating signal waveforms continuously is $20 \mathrm{MBaud}$ with roll-off factor 0.25 . In other words, when the spectrum of the generated communication signal exceeds the spectrum defined by the above symbol rate and in-excess bandwidth factor, the signal generation procedure is finite.

Focusing on the finite operational mode, platform memory resources comprise a key feature that affects the duration of 


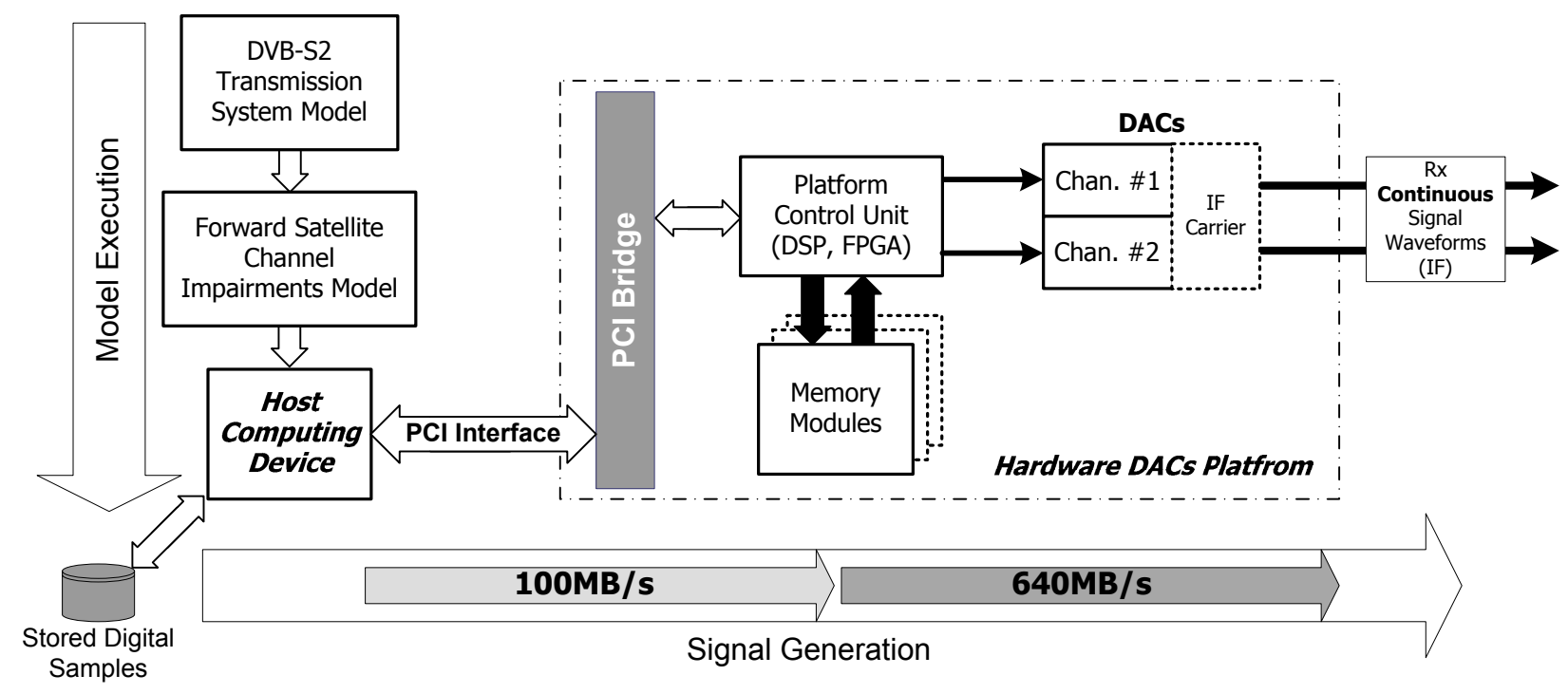

Fig. 4. Instrument's Architecture.

TABLE I

SUPPORTED DVB-S2 SyMBOL RATES (BASEBAND BANDWIDTH-MINIMUM SAMPLING RATES) VERSUS DAC OUTPUT Signal Durations (Finite Mode).

\begin{tabular}{|c|c|c|c|c|}
\hline \multirow{2}{*}{$\begin{array}{c}\text { Symbol } \\
\text { Rate } \\
\text { [MBaud] }\end{array}$} & \multirow{2}{*}{$\begin{array}{c}\text { Baseband } \\
\text { BW [MHz] } \\
\alpha=0.35\end{array}$} & \multirow{2}{*}{$\begin{array}{c}\text { Minimum } \\
\text { Sampling } \\
\text { Frequency [MSps] }\end{array}$} & \multicolumn{2}{|c|}{$\begin{array}{c}\text { DAC Output Signal } \\
\text { Duration }[\mathrm{Sec}]\end{array}$} \\
\hline & & & $16 \mathrm{MB}$ & $128 \mathrm{MB}$ \\
\hline 25 & 16.875 & 33.75 & 0.5371 & 4.2971 \\
\hline 35 & 23.625 & 47.25 & 0.2598 & 2.0782 \\
\hline 45 & 30.375 & 60.75 & 0.1919 & 1.5351 \\
\hline 55 & 37.125 & 74.25 & 0.1612 & 1.2897 \\
\hline
\end{tabular}

generated signal waveforms. In Table I, the supported symbol rates (of maximum roll-off factor 0.35 according to DVB-S2) along with the minimum required DACs sampling rates and the related output signal durations based on two setups of available platform memory are given. This mode of operation is used for generating a user-defined signal suitable for testing various receiver tasks such as receiver synchronization during initial acquisition (frame-carrier synchronization). Conventional synchronization techniques, usually used in typical satellite receiver designs, require over $100 \mathrm{msec}$ for initial acquisition convergence. In Figure 4 the instrument's internal architecture is presented.

As a further work we are planning to design a real-time forward satellite channel emulator which will be based on an advanced and powerful software radio platform that combines FPGA and DSP circuits.

\section{CONCLUSIONS}

In this work, a versatile instrument was presented for generating DVB-S2 compatible signal with the impairments en- countered in typical GEO forward satellite channels. The key feature of the proposed instrument is that it includes the impairments induced by the ODUs and frequency conversion stages of the entire processing chain of typical DVB-S2 communications systems, providing test signal under real conditions. Additionally, the ability of providing either analog waveforms or digital samples makes the instrument suitable for testing and evaluating a wide range of digital receiver implementations. By these means, newly developed DVB-S2 satellite receivers can be systematically and exhaustively validated and verified in terms of performance and functionality. Receiver realization designs and signal processing techniques can also be studied concerning their efficiency under DVB-S2 communications systems peculiarities acquiring interesting results for further analysis and improvement.

\section{REFERENCES}

[1] ETSI EN 302307 v1.1.1 (2005-03) Digital Video Broadcasting (DVB); Second generation framing structure, channel coding and modulation systems for Broadcasting, Interactive Services, News Gathering and other broadband satellite applications (DVB-S2).

[2] A. Morello and V. Mignone, "DVB-S2: The Second Generation Standard for Satellite Broad-band Services," Proc. IEEE, vol. 94, no. 1, pp. 210227, Jan. 2006

[3] G.E. Corazza M. Neri R. Pedone P. Salmi A. Vanelli-Coralli M. Villanti G. Albertazzi, S. Cioni, "On the Adaptive DVB-S2 Physical Layer: Design and Performance," IEEE Wireless Communications, pp. 62-68, DEC 2005.

[4] ETSI TR 102376 V1.1.1 (2005-02), Digital Video Broadcasting (DVB); User guidelines for the second generation system for Broadcasting, Interactive Services, News Gathering and other broadband satellite applications $(D V B-S 2)$.

[5] R. DeGaudenzi E. Casini and A. Ginesi, "DVB-S2 modem algorithms design and performance over typical satellite channels," International Journal of Satellite Communications and Networking 2004, vol. 22(3), pp. 281318, 2004.

[6] Dr. Kamilo Feher, Digital Communications: Satellite/Earth Station Engineering, Plenum Press, 1997.

[7] HELLAS-SAT 2 SATELLITE HANDBOOK, March 2004. 